\title{
A Practical Total Synthesis of (+)-Antimycin $\mathbf{A}_{\mathbf{9}}$
}

\author{
Takeshi Nishii, Makoto Inai, Hiroto Kaku, Mitsuyo Horikawa, Tetsuto Tsunoda
}

Received: October 2, 2006 / Accepted: December 21, 2006

(C) Japan Antibiotics Research Association

\begin{abstract}
Antimycin $\mathrm{A}_{9}\left(\mathrm{AA}_{9}\right)$ isolated from a cultured broth of Streptomyces sp. K01-0031 was synthesized via an asymmetric aldol reaction using Oppolzer's sultam as a chiral auxiliary.
\end{abstract}

Keywords asymmetric synthesis, asymmetric aldol reaction, antimycin $\mathrm{A}_{9}$, antibiotics

The antimycin A (AA) family isolated from Streptomyces sp. possesses significant biological activities [1], such as antifungal, insecticidal, and anticancer, as well as an inhibitory activity against ubiquinol-cytochrome $c$ oxidoreductase. Among the AA family, $\mathrm{AA}_{3}$ is one of the most active agents and has been widely used in biological and biochemical studies. On the contrary, $(+)-\mathrm{AA}_{9}$ (Figure 1) recently isolated from a cultured broth of Streptomyces sp. K01-0031 together $\mathrm{AA}_{3 \mathrm{a}}, \mathrm{AA}_{3 \mathrm{~b}}, \mathrm{AA}_{4}$, and $\mathrm{AA}_{7}$ by Kitasato's groups showed more potent nematocidal and insecticidal activities against Caenorhaditis elegans and Artemia salina than other known antimycins [1b]. Thus, involving the discovery of new compounds, investigation of the AA family is still continued because of their characteristic biological activities. For precise biological and biochemical investigations, the supply of each pure component of the AA family seems to be very important. However, since the isolation of each pure component from a cultured broth is possible only with intensive effort [2], the pure material of each component is not practically available for biological and biochemical studies. Therefore,

T. Tsunoda (Corresponding author), T. Nishii, M. Inai, H. Kaku, M. Horikawa: Faculty of Pharmaceutical Sciences, Tokushima Bunri University, Tokushima 770-8514, Japan, E-mail: tsunoda@ph.bunri-u.ac.jp the Antimycin A complex (mixture of AA family purchased from Sigma Co.) was used in most biological and biochemical studies.

Due to their outstanding bioactivities and unique structures, the antimycin family has attracted much interest from synthetic organic chemists [3]. Up to now three groups accomplished enantioselective total synthesis of $\mathrm{AA}_{3 \mathrm{~b}}$ (previous name $\mathrm{AA}_{3}$ ) [3b e]. However, the synthesis of other antimycins has not been performed and further effort directed to a practical and economical synthesis is still needed to supply sufficient amounts of pure antimycins for the biological investigations.

In the previous paper, we reported the synthesis of (+)$\mathrm{AA}_{3 \mathrm{a}}$ and (+)- $\mathrm{AA}_{3 \mathrm{~b}}$ using an asymmetric aza-Claisen rearrangement, although the overall yield was low [3c,d]. To develop a practical synthetic route of antimycins, we reconsidered the synthetic route and found that an asymmetric aldol reaction using Oppolzer's sultam as a chiral auxiliary was quite effective to construct the C-7 and C-8 asymmetric centers. Utilizing this methodology, we performed the first and efficient synthesis of $(+)-\mathrm{AA}_{9}$, which is the first antimycin having an aromatic 8-acyl residue. The results are reported herein.

Sultam amide 2 reacted with aldehyde 3 under the modified [4] Oppolzer's conditions to give the desired aldol adduct $\mathbf{4}$ and its stereoisomer [5] in $82 \%$ yield (ratio $=98 / 2$ )

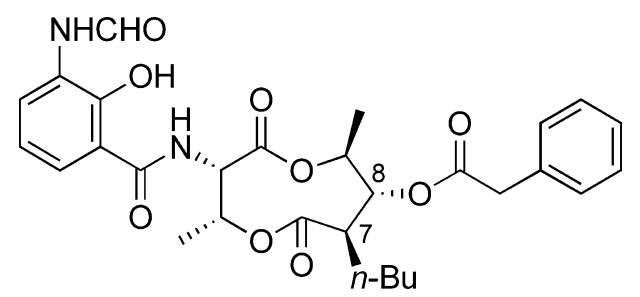

Fig. 1 Structure of antimycin $A_{9}$. 

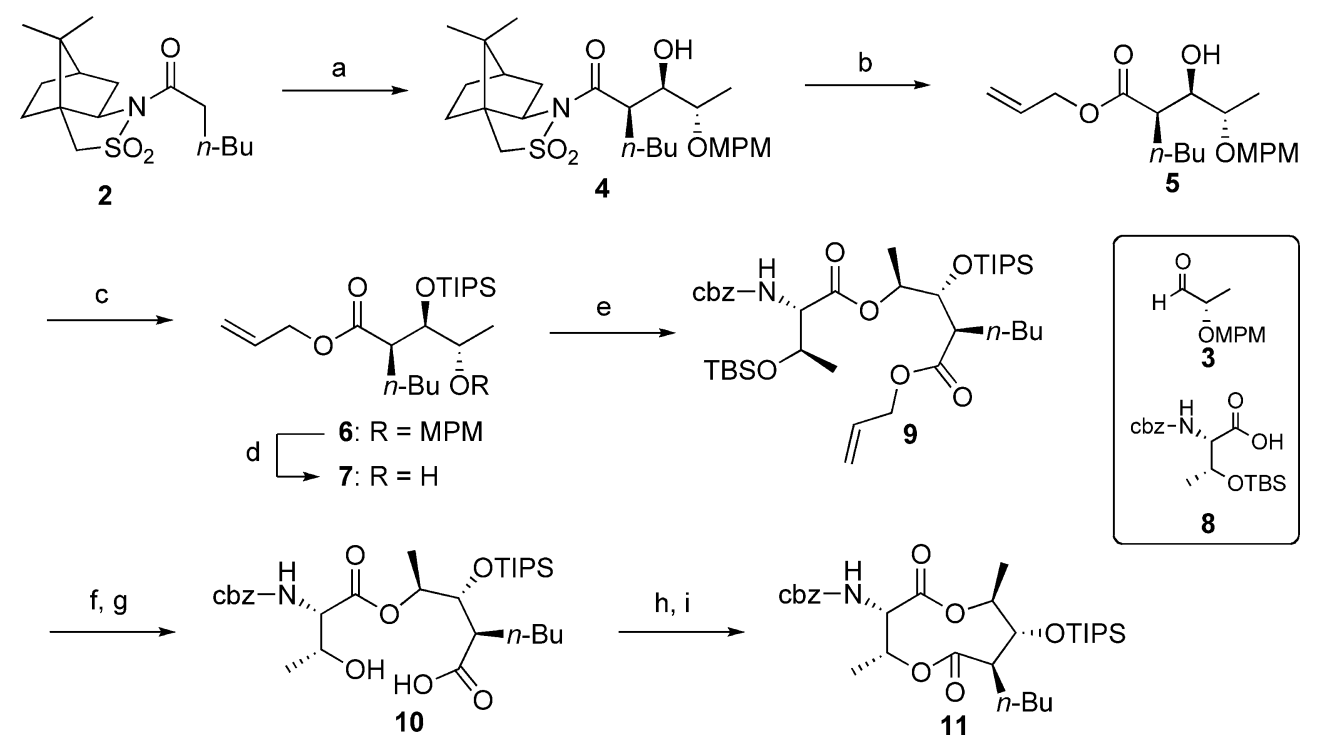

\section{Scheme 1}

Reagents and conditions: (a) (i) $n$ - $\mathrm{Bu}_{2} \mathrm{BOTf}$, DIPEA, $\mathrm{CH}_{2} \mathrm{Cl}_{2}, 0.5$ hours, $-5^{\circ} \mathrm{C}$; (ii) 3, 1 hour, $-78^{\circ} \mathrm{C}, 82 \%$ (98/2); (iii) recryst.; (b) Ti(O-iPr) 4 , MS 4A, allyl alcohol, $150^{\circ} \mathrm{C}, 48$ hours, 74\%; (c) TIPSOTf, DIPEA, $\mathrm{CH}_{2} \mathrm{Cl}_{2}$, r.t., 4 hours, 99\%; (d) $\mathrm{DDQ}, \mathrm{CH}_{2} \mathrm{Cl}_{2} / \mathrm{H}_{2} \mathrm{O}$, r.t., 0.5 hours; (e) (i) 8 , 2,4,6-trichlorobenzoyl chloride, $\mathrm{Et}_{3} \mathrm{~N}$, THF, r.t., 0.5 hours; (ii) DMAP, Tol, r.t., 15 hours, 2 steps $84 \%$; (f) $6 \mathrm{M} \mathrm{HCl,} \mathrm{EtOH,} \mathrm{r.t.,} 24$ hours, quant.; (g) $\mathrm{Pd}\left(\mathrm{PPh}_{3}\right)_{4}, \mathrm{PPh}_{3}$, pyrrolidine, $\mathrm{CH}_{3} \mathrm{CN}, 0^{\circ} \mathrm{C} \sim$ r.t., 7 hours; (h) 2,2'-dipyridyl disulfide, $\mathrm{PPh}_{3}$, Tol, r.t., 3 hours, 2 steps $91 \%$; (i) $(\mathrm{CuOTf})_{2} \cdot \mathrm{C}_{6} \mathrm{H}_{6}$, Tol, $80^{\circ} \mathrm{C}, 3$ hours, $87 \%$

[6]. The best results were obtained with 1.5 equivalents of dibutylboron trifluoromethanesulfonate $\left(n-\mathrm{Bu}_{2} \mathrm{BOTf}\right), 2.0$ equivalents of diisopropylethylamine (DIPEA), and 2.5 equivalents of $\mathbf{3}$. The pure $4(>99 \%$ de) afforded by recrystallization (63\% isolated yield) was converted to allyl ester 5 by heating at $150^{\circ} \mathrm{C}$ with allyl alcohol in the presence of titanium isopropoxide $\left[\mathrm{Ti}(\mathrm{O}-i \mathrm{Pr})_{4}\right]$ and molecular sieves $4 \AA$ (MS 4A) (74\%) [7].

After protection of the hydroxy group by triisopropylsilyl (TIPS) group, the $p$-methoxyphenylmethyl (MPM) group was removed with 2,3-dichloro-5,6-dicyano-1,4benzoquinone (DDQ) to yield alcohol 7. Condensation of 7 with L-threonine derivative 8 [8] proceeded satisfactorily under Yamaguchi conditions to give 9 in $84 \%$ yield (2 steps) [9]. Removal of the TBS group with $6 \mathrm{M}$ $\mathrm{HCl}$ followed by palladium(0)-catalyzed deprotection $\left[\mathrm{Pd}\left(\mathrm{PPh}_{3}\right)_{4}, \mathrm{PPh}_{3}\right.$, pyrrolidine] of the allyl ester provided the seco acid 10. Via conversion of $\mathbf{1 0}$ to the 2-pyridinethiol ester, lactonization was accomplished by heating at $80^{\circ} \mathrm{C}$ in toluene with copper(I) trifluoromethanesulfonate-benzene complex $\left[(\mathrm{CuOTf})_{2} \cdot \mathrm{PhH}\right]$ under high dilution conditions to give dilactone $\mathbf{1 1}$ in 79\% yield (4 steps from 9) [10].

The TIPS group of lactone $\mathbf{1 1}$ was removed smoothly using hydrogen fluoride pyridine (HF-Py) at r.t. to provide alcohol 12 (98\% yield), which was esterified with phenylacetic acid in the presence of $N$-(3-Dimethylaminopropyl)- $N$ '-ethylcarbodiimide hydrochloride [water- soluble carbodiimide (WSC)] and 4-( $N, N$-dimethylamino)pyridine (DMAP) in $\mathrm{CH}_{2} \mathrm{Cl}_{2}$ to provide ester 13 in excellent yield (92\%). The benzyloxycarbonyl (cbz) group of $\mathbf{1 3}$ was removed by hydrogenolysis (Pd-C in THF) to give an amine, which was successfully acylated with 14 (See the experimental section.), using WSC, 1-hydroxybenzotriazole hydrate (HOBt), and $N$-methylmorpholine (NMM) in DMF to give 15. Removal of the benzyl protecting group of $\mathbf{1 5}$ by hydrogenolysis with Pd-C in ethyl acetate led cleanly to the target molecule (+)-antimycin $\mathrm{A}_{9}(69 \%$ yield from 13), $\mathrm{mp}$ $151.0 \sim 152.0^{\circ} \mathrm{C},[\alpha]_{\mathrm{D}}^{22}+82.1\left(c 0.17, \mathrm{CH}_{3} \mathrm{OH}\right)$, whose physical properties compared well with those in the literature [1b] $\left[\mathrm{mp} 134 \sim 139^{\circ} \mathrm{C},[\alpha]_{\mathrm{D}}^{25}+83.6\right.$ (c 0.157 , $\left.\left.\mathrm{CH}_{3} \mathrm{OH}\right)\right]$.

Thus, the asymmetric aldol reaction using Oppolzer's sultam provided a practical and economical synthetic route (15 steps, overall yield $24 \%$ ) to (+)-antimycin $\mathrm{A}_{9}$, which could be obtained in pure form on a $300 \mathrm{mg}$ scale in this synthesis. Further synthetic studies of not only the AA family but also their analogs and investigation of their biological activities are now in progress. 


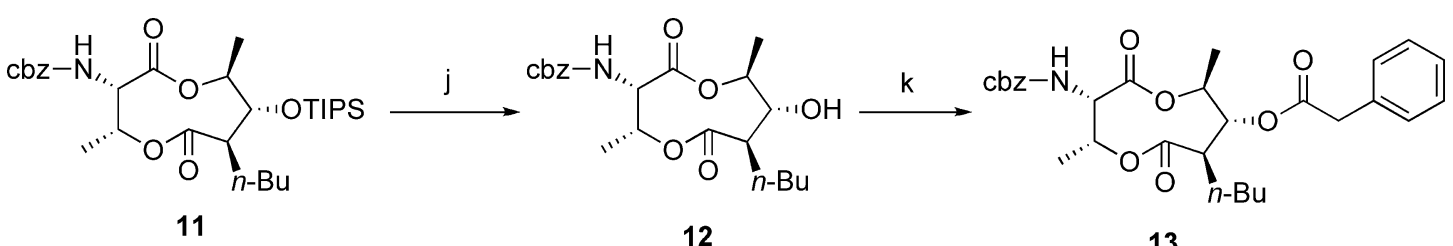

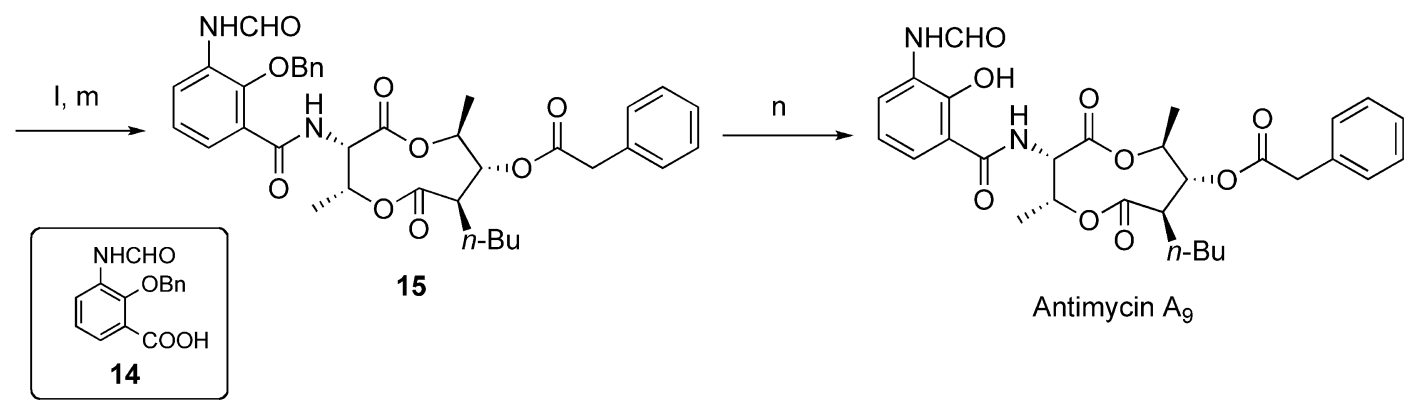

Scheme 2

Reagents and conditions: (j) HF.Py, THF, r.t., 3 hours, 98\%; (k) phenylacetic acid, WSC, DMAP, $\mathrm{CH}_{2} \mathrm{Cl}_{2}$, r.t., 1 hour, $92 \%$; (I) $\mathrm{H}_{2}$, $\mathrm{Pd} / \mathrm{C}$, THF, r.t., 2 hours; (m) 14, WSC, HOBt, NMM, DMF, r.t., 24 hours, 2 steps 77\%; (n) H2, Pd/C, EtOAc, r.t., 2 hours, $89 \%$.

\section{Experimental}

(3aS,6R,7aR)-1-\{2,5-Dideoxy-4-O-[(4-methoxyphenyl)methyl]-2-butyl-L-arabinoyl $\}$ hexahydro-8,8-dimethyl3H-3a,6-methano-2,1-benzisothiazole-2,2-dioxide (4)

To a suspension of $2(1.02 \mathrm{~g}, 3.25 \mathrm{mmol})$ in $\mathrm{CH}_{2} \mathrm{Cl}_{2}(10 \mathrm{ml})$ were added dibutylboron trifulate $\left(4.8 \mathrm{ml}, 1 \mathrm{M}\right.$ in $\mathrm{CH}_{2} \mathrm{Cl}_{2}$, $4.8 \mathrm{mmol})$ and DIPEA $(1.15 \mathrm{ml}, 6.6 \mathrm{mmol})$ at $-5^{\circ} \mathrm{C}$. After stirring at $-5^{\circ} \mathrm{C}$ for 0.5 hours and then cooling to $-78^{\circ} \mathrm{C}$, a solution of $3(1.58 \mathrm{~g}, 8.13 \mathrm{mmol})$ in $\mathrm{CH}_{2} \mathrm{Cl}_{2}(5 \mathrm{ml})$ was added dropwise to the mixture over 1 hour. The resulting mixture was treated with a phosphate buffer $(\mathrm{pH}$ 6.8) and saturated aqueous $\mathrm{NH}_{4} \mathrm{Cl}$, and the mixture was extracted with $\mathrm{CH}_{2} \mathrm{Cl}_{2}$. The organic extracts were dried over $\mathrm{MgSO}_{4}$, filtered, and concentrated. The residue $(5.22 \mathrm{~g})$ was purified by silica gel column chromatography (toluene/AcOEt) to give the aldol adduct $4(1.33 \mathrm{~g}, 82 \%)$ as colorless needles; $\mathrm{mp} 102.1 \sim 103.2^{\circ} \mathrm{C}$ (hexane/EtOAc); $[\alpha]_{\mathrm{D}}^{22}-48.8$ (c 1.0, $\mathrm{CHCl}_{3}$ ); IR (neat) $\mathrm{cm}^{-1}: 3509,2958,1686,1512,1333 ;{ }^{1} \mathrm{H}$ NMR $\left(300 \mathrm{MHz}, \mathrm{CDCl}_{3}\right) \delta: 7.29(2 \mathrm{H}, \mathrm{d}, J=8.5 \mathrm{~Hz}), 6.87$ $(2 \mathrm{H}, \mathrm{ddd}, J=9.5,0.4,4.8 \mathrm{~Hz}), 4.57(1 \mathrm{H}, \mathrm{d}, J=11.5 \mathrm{~Hz})$, $4.39(1 \mathrm{H}, \mathrm{d}, J=11.3 \mathrm{~Hz}), 3.97(1 \mathrm{H}$, ddd, $J=6.4,4.9$, $1.9 \mathrm{~Hz}), 3.86(1 \mathrm{H}, \mathrm{t}, J=6.3 \mathrm{~Hz}), 3.80(3 \mathrm{H}, \mathrm{s}), 3.57 \sim 3.41$ $(4 \mathrm{H}, \mathrm{m}), 2.87(1 \mathrm{H}, \mathrm{d}, J=1.6 \mathrm{~Hz}), 2.05(2 \mathrm{H}, \mathrm{d}, J=6.3 \mathrm{~Hz})$, $1.94 \sim 1.80(3 \mathrm{H}, \mathrm{m}), 1.39 \sim 1.18(11 \mathrm{H}, \mathrm{m}), 1.17(3 \mathrm{H}, \mathrm{s})$, $0.98(3 \mathrm{H}, \mathrm{s}), 0.83(3 \mathrm{H}, \mathrm{t}, J=6.8 \mathrm{~Hz})$; MS (EI) $\mathrm{m} / \mathrm{z} 507$ $\left(\mathrm{M}^{+}\right), 353,342,257,216,121$ (base peak); HRMS (EI) $\mathrm{m} / \mathrm{z}$ 507.2661 (507.2655 calcd for $\mathrm{C}_{27} \mathrm{H}_{41} \mathrm{O}_{6} \mathrm{NS}$ ).
(R)-Allyl-2-[(1R,2S)-1-hydroxy-2-(4-methoxybenzyloxy)propyl]hexanoate (5)

To a suspension of $4(202 \mathrm{mg}, 0.4 \mathrm{mmol})$ and $4 \AA \mathrm{MS}$ $(386 \mathrm{mg})$ in allyl alcohol $(4 \mathrm{ml})$ was added $\mathrm{Ti}(i-\mathrm{Pro})_{4}$ $(300 \mu \mathrm{l}, 1.2 \mathrm{mmol})$ at r.t., and the mixture was heated at $150^{\circ} \mathrm{C}$ for 48 hours. The resulting mixture was treated with saturated aqueous $\mathrm{NH}_{4} \mathrm{Cl}$ and then filtered through a pad of Celite. The filtrate was extracted with AcOEt, and the extracts were dried over $\mathrm{MgSO}_{4}$, filtered, and concentrated. The residue $(302 \mathrm{mg}$ ) was purified by silica gel column chromatography (hexane/EtOAc) to give the allyl ester $\mathbf{5}$ $(104 \mathrm{mg}, 74 \%)$ as a colorless oil; $[\alpha]_{\mathrm{D}}^{21}+27.3(c$ 1.0, $\mathrm{CHCl}_{3}$ ); IR (ATR) $\mathrm{cm}^{-1}: 3502,2955,2360,1728,1612$, 1586, 1512, 1172, 1073, 1034; ${ }^{1} \mathrm{H}$ NMR $\left(300 \mathrm{MHz}, \mathrm{CDCl}_{3}\right)$ $\delta 7.24(2 \mathrm{H}, \mathrm{dt}, J=9.0,2.7 \mathrm{~Hz}), 6.88(2 \mathrm{H}, \mathrm{dt}, J=9.0$, $2.7 \mathrm{~Hz}), 5.87(1 \mathrm{H}, \mathrm{ddt}, J=17.1,10.2,6.0 \mathrm{~Hz}), 5.39(1 \mathrm{H}$, $\mathrm{ddt}, J=17.1,1.5,1.5 \mathrm{~Hz}), 5.23(1 \mathrm{H}$, ddt, $J=10.2,1.2$, $1.2 \mathrm{~Hz}), 4.55(2 \mathrm{H}, \mathrm{ddd}, J=6.0,1.2,1.2 \mathrm{~Hz}), 4.51(1 \mathrm{H}, \mathrm{d}$, $J=11.4 \mathrm{~Hz}), 4.39(1 \mathrm{H}, \mathrm{d}, J=11.1 \mathrm{~Hz}), 3.88(1 \mathrm{H}, \mathrm{dd}, J=6.9$, $5.1 \mathrm{~Hz}), 3.80(3 \mathrm{H}, \mathrm{s}), 3.45(1 \mathrm{H}, \mathrm{qd}, J=5.1,0.9 \mathrm{~Hz}), 2.58$ $(1 \mathrm{H}, \mathrm{ddd}, J=10.2,6.9,4.5 \mathrm{~Hz}), 1.54 \sim 1.82(2 \mathrm{H}, \mathrm{m}), 1.21$ $(3 \mathrm{H}, \mathrm{d}, J=6.0 \mathrm{~Hz}), 1.18 \sim 1.36(4 \mathrm{H}, \mathrm{m}), 0.88(3 \mathrm{H}, \mathrm{t}$, $J=6.6 \mathrm{~Hz}) ;{ }^{13} \mathrm{C}$ NMR $\left(75 \mathrm{MHz}, \mathrm{CDCl}_{3}\right) \delta 174.7,159.2$, $131.9,130.2,129.4,118.5,113.7,74.7,73.4,70.0,65.0$, 55.2, 47.6, 29.5, 27.8, 22.6, 14.1, 13.9; MS (CI) $\mathrm{m} / z 350$ $\left(\mathrm{M}^{+}\right), 288,243,213,196,185,163,121$ (base peak); HRMS (CI) $m / z 350.2086\left(\mathrm{M}^{+}\right)(350.2093$ calcd for $\mathrm{C}_{20} \mathrm{H}_{30} \mathrm{O}_{5}$ ). 
(R)-Allyl-2-[(1R,2S)-2-(4-methoxybenzyloxy)-1(triisopropylsilyloxy)propyl]hexanoate (6)

To a solution of $5(66.8 \mathrm{mg}, 0.19 \mathrm{mmol})$ in $\mathrm{CH}_{2} \mathrm{Cl}_{2}(1 \mathrm{ml})$ were added DIPEA $(100 \mu \mathrm{l}, 0.57 \mathrm{mmol})$ and TIPSOTf $(104 \mu 1,0.39 \mathrm{mmol})$ successively at $0^{\circ} \mathrm{C}$. After stirring at r.t. for 4 hours, the reaction mixture was quenched with the addition of saturated aqueous $\mathrm{NH}_{4} \mathrm{Cl}$, and the resulting mixture was extracted with ether. The extracts were dried over $\mathrm{MgSO}_{4}$, filtered, and concentrated. The residue $(142 \mathrm{mg}$ ) was purified by silica gel column chromatography (hexane/AcOEt) to give the TIPS ether $6(95.4 \mathrm{mg}, 99 \%)$ as a colorless oil; $[\alpha]_{\mathrm{D}}^{24}+10.2\left(c 1.0, \mathrm{CHCl}_{3}\right)$; IR (ATR) $\mathrm{cm}^{-1}: 2943,1732,1613,1512,1463,1247,1106,1034 ;{ }^{1} \mathrm{H}$ NMR $\left(300 \mathrm{MHz}, \mathrm{CDCl}_{3}\right) \delta 7.22(2 \mathrm{H}, \mathrm{dt}, J=9.0,2.7 \mathrm{~Hz})$, $6.85(2 \mathrm{H}, \mathrm{dt}, J=8.7,3.0 \mathrm{~Hz}), 5.85(1 \mathrm{H}$, ddt, $J=17.1,10.2$, $6.0 \mathrm{~Hz}), 5.29(1 \mathrm{H}$, ddt, $J=17.4,1.8,1.2 \mathrm{~Hz}), 5.20(1 \mathrm{H}$, ddt, $J=10.5,1.5,1.2 \mathrm{~Hz}), 4.50(2 \mathrm{H}, \mathrm{dd}, J=5.7,1.2 \mathrm{~Hz}), 4.44$ $(1 \mathrm{H}, \mathrm{d}, J=11.7 \mathrm{~Hz}), 4.37(1 \mathrm{H}, \mathrm{d}, J=11.1 \mathrm{~Hz}), 4.13(1 \mathrm{H}, \mathrm{dd}$, $J=7.2,2.7 \mathrm{~Hz}), 3.80(3 \mathrm{H}, \mathrm{s}), 3.40(1 \mathrm{H}, \mathrm{qd}, J=6.3,2.7 \mathrm{~Hz})$, 2.49 (1H, ddd, $J=11.1,7.5,3.6 \mathrm{~Hz}), 1.57 \sim 1.82(2 \mathrm{H}, \mathrm{m})$, $1.17(3 \mathrm{H}, \mathrm{d}, J=6.3 \mathrm{~Hz}), 1.05 \sim 1.32(24 \mathrm{H}, \mathrm{m}), 0.87(3 \mathrm{H}, \mathrm{t}$, $J=6.9 \mathrm{~Hz}) ;{ }^{13} \mathrm{C} \mathrm{NMR}\left(75 \mathrm{MHz}, \mathrm{CDCl}_{3}\right) \delta 174.4,158.9$, $132.1,130.8,129.2,118.2,113.5,76.4,70.1,64.9,55.2$, 50.5, 30.0, 29.0, 22.6, 18.3, 14.0, 13.1; MS (CI) $\mathrm{m} / \mathrm{z} 507$ $\left([\mathrm{M}+\mathrm{H}]^{+}\right), 463,399,369,3269,241,163,121$ (base peak); HRMS (CI) $\mathrm{m} / z 507.3514\left([\mathrm{M}+\mathrm{H}]^{+}\right)(507.3505$ calcd for $\mathrm{C}_{29} \mathrm{H}_{51} \mathrm{O}_{5} \mathrm{Si}$ ).

\section{(R)-Allyl-2-\{(5S,8S,9R)-5-[(R)-1-(tert-}

butyldimethylsilyloxy)ethyl]-11,11-diisopropyl-8,12dimethyl-3,6-dioxo-1-phenyl-2,7,10-trioxa-4-aza-11silatridecan-9-yl\}hexanoate (9)

\section{Deprotection of $\mathbf{6}$}

To a solution of $6(2.01 \mathrm{~g}, 3.97 \mathrm{mmol})$ in $\mathrm{CH}_{2} \mathrm{Cl}_{2}(40 \mathrm{ml})$ were added dist. water $(2.2 \mathrm{ml})$ and DDQ $(988 \mathrm{mg}$, $4.4 \mathrm{mmol}$ ) at r.t., and the mixture was stirred for 0.5 hours at same temperature. The reaction mixture was quenched with the addition of saturated aqueous $\mathrm{NaHCO}_{3}$, and the resulting mixture was extracted with $\mathrm{CH}_{2} \mathrm{Cl}_{2}$. The organic extracts were dried over $\mathrm{MgSO}_{4}$, filtered, and concentrated to give the crude deprotected alcohol of $7(1.54 \mathrm{~g})$.

Preparation of a Mixed Anhydride of $\mathbf{8}$

To a solution of $8(2.91 \mathrm{~g}, 7.93 \mathrm{mmol})$ in THF $(15 \mathrm{ml})$ were added $\mathrm{NEt}_{3}(2.2 \mathrm{ml}, 16 \mathrm{mmol})$, and 2,4,6-trichlorobenzoyl chloride $(1.8 \mathrm{ml}, 12 \mathrm{mmol})$ at $0^{\circ} \mathrm{C}$. After stirring at r.t. for 1.5 hours, the resulting mixture was filtered and concentrated to give a mixed anhydride of $\mathbf{8}$.
Condensation of the Alcohol and the Mixed Anhydride

To a solution of the crude alcohol in toluene $(20 \mathrm{ml})$ were added DMAP (781 mg, $6.4 \mathrm{mmol}), \mathrm{NEt}_{3}(550 \mu \mathrm{l}$, $3.95 \mathrm{mmol}$ ), and a solution of the mixed anhydride in toluene $(20 \mathrm{ml})$ successively at $0^{\circ} \mathrm{C}$. The resulting mixture was stirred at r.t. for 16 hours and quenched with the addition of dist. water. The resulting mixture was extracted with ether, and the organic extracts were washed with brine, dried over $\mathrm{MgSO}_{4}$, filtered, and concentrated. The residue $(5.36 \mathrm{~g})$ was purified by silica gel column chromatography (hexane/EtOAc) to give the diester 9 (2.44 g, 84\%, 2 steps) as a colorless oil; $[\alpha]_{\mathrm{D}}^{23}+0.52\left(c 1.0, \mathrm{CHCl}_{3}\right)$; IR (ATR) $\mathrm{cm}^{-1}: 2946,2865,1730,1504,1463,1378,1311,1252$, 1170,$1103 ;{ }^{1} \mathrm{H}$ NMR $\left(300 \mathrm{MHz}, \mathrm{CDCl}_{3}\right) \delta 7.24 \sim 7.44(5 \mathrm{H}$, m), $5.91(1 \mathrm{H}, \mathrm{ddt}, J=17.1,10.2,6.0 \mathrm{~Hz}), 5.44(1 \mathrm{H}, \mathrm{brd}$, $J=9.6 \mathrm{~Hz}), 5.32(2 \mathrm{H}$, ddt, $J=17.1,1.5,1.5 \mathrm{~Hz}), 5.24(1 \mathrm{H}$, ddt, $J=10.5,1.2,1.2 \mathrm{~Hz}), 5.19(1 \mathrm{H}, \mathrm{d}, J=12.0 \mathrm{~Hz}), 5.06$ $(1 \mathrm{H}, \mathrm{d}, J=12.0 \mathrm{~Hz}), 4.84(1 \mathrm{H}, \mathrm{qd}, J=6.3,1.8 \mathrm{~Hz})$, $4.49 \sim 4.65(2 \mathrm{H}, \mathrm{m}), 4.40(1 \mathrm{H}, \mathrm{qd}, J=6.0,1.5 \mathrm{~Hz}), 4.19$ (1H, dd, $J=9.9,1.5 \mathrm{~Hz}), 4.13(1 \mathrm{H}, \mathrm{dd}, J=7.5,1.8 \mathrm{~Hz}), 2.48$ $(1 \mathrm{H}$, ddd, $J=10.5,7.5,3.9 \mathrm{~Hz}), 1.60 \sim 1.80(2 \mathrm{H}, \mathrm{m}), 1.27$ $(3 \mathrm{H}, \mathrm{d}, J=6.3 \mathrm{~Hz}), 1.19(3 \mathrm{H}, \mathrm{d}, J=6.0 \mathrm{~Hz}), 1.02 \sim 1.38$ $(25 \mathrm{H}, \mathrm{m}), 0.88(3 \mathrm{H}, \mathrm{t}, J=6.6 \mathrm{~Hz}), 0.80 \sim 0.92(9 \mathrm{H}, \mathrm{m})$, $-0.08 \sim 0.06(6 \mathrm{H}, \mathrm{m}) ;{ }^{13} \mathrm{C} \mathrm{NMR}\left(75 \mathrm{MHz}, \mathrm{CDCl}_{3}\right) \delta 173.5$, $170.0,156.5,136.2,131.2,128.5,128.1,118.7,75.8,74.5$, $68.8,67.1,65.2,59.9,50.7,29.8,29.2,25.6,22.5,21.1$, $18.2,17.8,13.8,13.5,13.0,-4.5,-5.1$; MS (FAB) $\mathrm{m} / \mathrm{z}$ $759\left([\mathrm{M}+\mathrm{Na}]^{+}\right), 678,544,480,448,369,241,91$ (base peak); HRMS (FAB) $m / z 758.4465\left([\mathrm{M}+\mathrm{Na}]^{+}\right)(758.44591$ calcd for $\mathrm{C}_{39} \mathrm{H}_{69} \mathrm{NO}_{8} \mathrm{Si}_{2} \mathrm{Na}$ ).

\section{Benzyl-(3S,4R,7R,8R,9S)-7-butyl-4,9-dimethyl-2,6- dioxo-8-(triisopropylsilyloxy)-1,5-dioxonan-3- ylcarbamate (10)}

\section{Desilylation of 9}

To a solution of $9(125 \mathrm{mg}, 0.17 \mathrm{mmol})$ in EtOH $(1.5 \mathrm{ml})$ was added $6 \mathrm{M} \mathrm{HCl}(185 \mu \mathrm{l})$ at r.t., and the mixture was stirred for 24 hours. The reaction was quenched with the addition of saturated aqueous $\mathrm{NaHCO}_{3}$. The resulting mixture was extracted with ether, and the organic extracts were washed with brine, dried over $\mathrm{MgSO}_{4}$, filtered, and concentrated. The residue $(119 \mathrm{mg})$ was purified by silica gel column chromatography (hexane/ether) to give a desilylated alcohol (105 mg, quant.) as a colorless oil; $[\alpha]_{D}^{22}$ -12.1 (c 1.0, $\mathrm{CHCl}_{3}$ ); IR (ATR) $\mathrm{cm}^{-1}: 3444,2944,2867$, $1729,1515,1456,1382 ;{ }^{1} \mathrm{H}$ NMR $\left(300 \mathrm{MHz}, \mathrm{CDCl}_{3}\right) \delta$ $7.28 \sim 7.42$ (5H, m), 5.91 (1H, ddt, $J=17.1,10.2,6.0 \mathrm{~Hz})$, $5.53(1 \mathrm{H}$, br d, $J=12.6 \mathrm{~Hz}), 5.33(1 \mathrm{H}, \mathrm{ddt}, J=17.1,1.5$, $1.5 \mathrm{~Hz}), 5.25(1 \mathrm{H}, \mathrm{ddt}, J=10.5,1.2,1.2 \mathrm{~Hz}), 5.17(1 \mathrm{H}, \mathrm{d}$, $J=12.6 \mathrm{~Hz}), 5.09(1 \mathrm{H}, \mathrm{d}, J=12.0 \mathrm{~Hz}), 4.90 \sim 5.05(1 \mathrm{H}, \mathrm{m})$, 
$4.58(2 \mathrm{H}$, ddd, $J=6.0,1.2,1,2 \mathrm{~Hz}), 4.22 \sim 4.42(2 \mathrm{H}, \mathrm{m})$, $4.10(1 \mathrm{H}, \mathrm{dd}, J=6.0,3.6 \mathrm{~Hz}) 2.46 \sim 2.66(1 \mathrm{H}, \mathrm{m})$, $1.62 \sim 1.82(2 \mathrm{H}, \mathrm{m}), 1.26(3 \mathrm{H}, \mathrm{d}, J=6.3 \mathrm{~Hz}), 1.24(3 \mathrm{H}, \mathrm{d}$, $J=6.3 \mathrm{~Hz}), 1.02 \sim 1.40(25 \mathrm{H}, \mathrm{m}), 0.88(3 \mathrm{H}, \mathrm{t}, J=6.9 \mathrm{~Hz})$; ${ }^{13} \mathrm{C}$ NMR $\left(75 \mathrm{MHz}, \mathrm{CDCl}_{3}\right) \delta 174.0,170.2,156.5,136.1$, $131.8,128.4,128.0,127.9,118.7,75.9,74.7,67.7,67.0$, 65.3, 59.3, 50.4, 29.9, 29.0, 22.5, 19.6, 18.1, 15.3, 13.8, 12.9; MS (CI) $m / z 622\left([\mathrm{M}+\mathrm{H}]^{+}\right), 578,514,470,369$ (base peak), 329, 285, 241, 195, 131, 91; HRMS (CI) m/z $622.3759\left([\mathrm{M}+\mathrm{H}]^{+}\right)\left(622.3774\right.$ calcd for $\left.\mathrm{C}_{33} \mathrm{H}_{56} \mathrm{NO}_{8} \mathrm{Si}\right)$.

\section{Preparation of Seco Acid 10}

To a solution of the desilylated alcohol $(81.2 \mathrm{mg}$, $0.13 \mathrm{mmol})$ in acetonitrile $(1 \mathrm{ml})$ were added $\mathrm{Pd}\left(\mathrm{PPh}_{3}\right)_{4}$ (4.2 mg, $3.6 \mu \mathrm{mol}), \mathrm{PPh}_{3}(1.8 \mathrm{mg}, 6.7 \mu \mathrm{mol})$ and pyrrolidine $(10 \mu \mathrm{l}, 0.15 \mathrm{mmol})$ successively at $0^{\circ} \mathrm{C}$. Since the alcohol still remained after stirring at r.t. for 3 hours (monitored by TLC), the same amount of the reagents were added to the mixture. After additional stirring for 4 hours, the resulting mixture was treated with $15 \% \mathrm{HCl}$ aq. and $\mathrm{NaCl}$. The resulting was extracted with $\mathrm{CH}_{2} \mathrm{Cl}_{2}$, and the organic extracts were dried over $\mathrm{MgSO}_{4}$, filtered, and concentrated. The residue $(110 \mathrm{mg})$ was purified by silica gel column chromatography (hexane/acetone) to give the crude seco acid $\mathbf{1 0}(78.8 \mathrm{mg})$.

\section{Conversion of $\mathbf{1 0}$ to Pyridinethiol Ester}

To a solution of the crude seco acid $\mathbf{1 0}$ in toluene $(1 \mathrm{ml})$ were added $\mathrm{PPh}_{3}(131 \mathrm{mg}, 0.595 \mathrm{mmol})$ and PySSPy $(156 \mathrm{mg}, 0.6 \mathrm{mmol})$ at r.t., and the mixture was stirred for 3 hours. After concentration the residue was purified by silica gel column chromatography (hexane/acetone) to give the pyridinethiol ester $(81.5 \mathrm{mg}, 91 \%, 2$ steps $)$ as a pale yellow oil; $[\alpha]_{\mathrm{D}}^{22}-22.3$ (c 1.0, $\mathrm{CHCl}_{3}$ ); IR (ATR) $\mathrm{cm}^{-1}: 3375$, 2943, 2866, 2360, 1698, 1511, 1454, 1421, 1318, 1201, $1164,1116,1055 ;{ }^{1} \mathrm{H}$ NMR $\left(300 \mathrm{MHz}, \mathrm{CDCl}_{3}\right) \delta$ $8.54 \sim 8.62(1 \mathrm{H}, \mathrm{m}), 7.75(1 \mathrm{H}, \mathrm{td}, J=7.5,1.8 \mathrm{~Hz}), 7.61(1 \mathrm{H}$, d, $J=9.0 \mathrm{~Hz}), \quad 7.24 \sim 7.42(6 \mathrm{H}, \quad \mathrm{m}), \quad 5.70(1 \mathrm{H}, \quad$ br d, $J=9.7 \mathrm{~Hz}), \quad 5.04 \sim 5.22(3 \mathrm{H}, \mathrm{m}), 4.04 \sim 4.46(3 \mathrm{H}, \mathrm{m})$, $2.74 \sim 3.24(2 \mathrm{H}, \mathrm{m}), 1.68 \sim 1.92(2 \mathrm{H}, \mathrm{m}), 1.34(3 \mathrm{H}, \mathrm{brd}$, $J=6.3 \mathrm{~Hz}), 1.23(3 \mathrm{H}, \mathrm{d}, J=6.3 \mathrm{~Hz}), 1.02 \sim 1.48(25 \mathrm{H}, \mathrm{m})$, $0.90(3 \mathrm{H}, \mathrm{t}, J=6.9 \mathrm{~Hz}) ;{ }^{13} \mathrm{C} \mathrm{NMR}\left(75 \mathrm{MHz}, \mathrm{CDCl}_{3}\right) \delta$ $198.5,170.4,156.5,151.1,150.2,137.2,136.2,130.1$, 128.4, 128.1, 127.9, 123.6, 76.1, 74.6, 67.6, 67.0, 59.3, $59.1,31.5,29.6,29.3,27.6,22.5,19.8,18.2,15.4,14.0$, 13.0; MS (CI) $m / z 675\left([\mathrm{M}+\mathrm{H}]^{+}\right), 567,520,419,366,311$, 283, 245, 208, 155, 112 (base peak), 91; HRMS (CI) $\mathrm{m} / \mathrm{z}$ $675.3483\left([\mathrm{M}+\mathrm{H}]^{+}\right)\left(675.3499\right.$ calcd for $\left.\mathrm{C}_{35} \mathrm{H}_{55} \mathrm{~N}_{2} \mathrm{O}_{7} \mathrm{SSi}\right)$.

Lactonization

To a warmed solution of $(\mathrm{CuOTf})_{2} \cdot \mathrm{C}_{6} \mathrm{H}_{6} \quad(561 \mathrm{mg}$,
$1.0 \mathrm{mmol}$ ) in toluene (1 liter) at $80^{\circ} \mathrm{C}$ was added dropwise a solution of the pyridinethiol ester $(657 \mathrm{mg}, 0.97 \mathrm{mmol})$ in toluene $(20 \mathrm{ml})$ over 2 hours. The resulting mixture was stirred for 1 hour at same temperature and filtered through a pad of silica gel. The filtrate was concentrated, and the residue $(913 \mathrm{mg})$ was purified by silica gel column chromatography (hexane/AcOEt) to give the 9-membered dilactone $11(476 \mathrm{mg}, 87 \%)$ as a colorless oil; $[\alpha]_{\mathrm{D}}^{24}+36.3$ (c 1.0, $\mathrm{CHCl}_{3}$ ); IR (ATR) $\mathrm{cm}^{-1}: 2944,2867,2360,1740$, $1509,1103,1057,1013 ;{ }^{1} \mathrm{H}$ NMR $\left(300 \mathrm{MHz}, \mathrm{CDCl}_{3}\right) \delta$ $7.24 \sim 7.46(5 \mathrm{H}, \mathrm{m}), 5.71(1 \mathrm{H}, \mathrm{brd}, J=7.8 \mathrm{~Hz}), 5.53(1 \mathrm{H}$, quintet, $J=6.9 \mathrm{~Hz}), 5.11(2 \mathrm{H}, \mathrm{s}), 4.95(1 \mathrm{H}, \mathrm{t}, J=8.1 \mathrm{~Hz})$, $4.76(1 \mathrm{H}$, quintet, $J=6.0 \mathrm{~Hz}), 3.83(1 \mathrm{H}, \mathrm{t}, J=8.7 \mathrm{~Hz}), 2.31$ $(1 \mathrm{H}, \mathrm{dt}, J=14.7,9.3 \mathrm{~Hz}), 1.58 \sim 1.82(2 \mathrm{H}, \mathrm{m}), 1.39(3 \mathrm{H}, \mathrm{d}$, $J=6.3 \mathrm{~Hz}), 1.25(3 \mathrm{H}, \mathrm{d}, J=6.9 \mathrm{~Hz}), 1.02 \sim 1.42(23 \mathrm{H}, \mathrm{m})$, $0.88(3 \mathrm{H}, \mathrm{t}, J=6.9 \mathrm{~Hz}) ;{ }^{13} \mathrm{C}$ NMR $\left(75 \mathrm{MHz}, \mathrm{CDCl}_{3}\right) \delta$ $174.1,170.1,155.4,135.9,128.4,128.1,127.9,78.9,76.8$, 70.7, 67.1, 54.7, 53.2, 29.6, 29.0, 22.4, 18.5, 18.1, 14.7, 13.7; MS (CI) $m / z 564\left([\mathrm{M}+\mathrm{H}]^{+}\right), 520,456,419$ (base peak), 329, 278, 236; HRMS (CI) $\mathrm{m} / z 564.3342\left([\mathrm{M}+\mathrm{H}]^{+}\right)$ (564.3356 calcd for $\mathrm{C}_{30} \mathrm{H}_{50} \mathrm{NO}_{7} \mathrm{Si}$ ).

\section{Benzyl-(3S,4R,7R,8R,9S)-7-butyl-8-hydroxy-4,9- dimethyl-2,6-dioxo-1,5-dioxonan-3-ylcarbamate (12)}

To a solution of $11(449 \mathrm{mg}, 0.80 \mathrm{mmol})$ in THF $(4 \mathrm{ml})$ was added HF.pyridine $(4 \mathrm{ml})$ at r.t. After stirring at r.t. for 3 hours, the mixture was poured into saturated aqueous $\mathrm{NaHCO}_{3}$, and the resulting mixture was extracted with EtOAc. The oreganic extracts were dried over $\mathrm{MgSO}_{4}$, filtered, and concentrated. The residue $(414 \mathrm{mg})$ was purified by silica gel column chromatography (hexane/ EtOAc) to give the alcohol $12(320 \mathrm{mg}, 98 \%)$ as colorless needles; mp 104.5 105. $0^{\circ} \mathrm{C}$ (hexane/EtOAc); $[\alpha]_{\mathrm{D}}^{23}+47.4$ (c 1.0, $\mathrm{CHCl}_{3}$ ); IR (ATR) $\mathrm{cm}^{-1}: 3360,2956,1701,1519$, 1361, 1267, 1227, 1177, 1045; ${ }^{1} \mathrm{H}$ NMR $\left(300 \mathrm{MHz}, \mathrm{CDCl}_{3}\right)$ $\delta 7.28 \sim 7.42(5 \mathrm{H}, \mathrm{m}), 5.42 \sim 5.64(2 \mathrm{H}, \mathrm{m}), 5,11(2 \mathrm{H}, \mathrm{s})$, $4.92(1 \mathrm{H}, \mathrm{t}, J=8.4 \mathrm{~Hz}), 4.68 \sim 4.85(1 \mathrm{H}, \mathrm{m}), 3.44 \sim 3.62$ $(1 \mathrm{H}, \mathrm{m}), 2.30(1 \mathrm{H}$, ddd, $J=11.1,9.6,3.3 \mathrm{~Hz}), 2.24 \sim 2.42$ $(1 \mathrm{H}, \mathrm{m}), 1.58 \sim 1.86(2 \mathrm{H}, \mathrm{m}), 1.41(3 \mathrm{H}, \mathrm{d}, J=6.3 \mathrm{~Hz}), 1.27$ $(3 \mathrm{H}$, br d, $J=5.7 \mathrm{~Hz}), 1.14 \sim 1.36(4 \mathrm{H}, \mathrm{m}), 0.88(3 \mathrm{H}, \mathrm{t}$, $J=6.9 \mathrm{~Hz}) ;{ }^{13} \mathrm{C}$ NMR $\left(75 \mathrm{MHz}, \mathrm{CDCl}_{3}\right) \delta 174.1,170.3$, 155.6, 135.8, 128.5, 128.3, 128.0, 76.2, 70.8, 67.3, 54.9, 52.0, 29.4, 28.6, 22.5, 18.3, 14.9, 13.8; MS (CI) $\mathrm{m} / \mathrm{z} 408$ $\left([\mathrm{M}+\mathrm{H}]^{+}\right), 390,364,300,263,236,208,173,91$ (base peak); HRMS (CI) $\mathrm{m} / \mathrm{z} 408.2010\left([\mathrm{M}+\mathrm{H}]^{+}\right)(408.2022$ calcd for $\mathrm{C}_{21} \mathrm{H}_{30} \mathrm{NO}_{7}$ ).

(2R,3S,6S,7R,8R)-3-(Benzyloxycarbonylamino)-8-butyl2,6-dimethyl-4,9-dioxo-1,5-dioxonan-7-yl 2-Phenylethanoate (13)

To a solution of $12(334 \mathrm{mg}, 0.82 \mathrm{mmol})$ in $\mathrm{CH}_{2} \mathrm{Cl}_{2}$ were 
added phenyl acetic acid $(227 \mathrm{mg}, 1.7 \mathrm{mmol})$, WSC (240 mg, $1.3 \mathrm{mmol}$ ) and DMAP (50.9 mg, $0.42 \mathrm{mmol}$ ) successively at r.t. The mixture was stirred for 1 hour and treated with saturated aqueous $\mathrm{NaHCO}_{3}$. The resulting mixture was extracted with AcOEt, and the organc extracts were dried over $\mathrm{MgSO}_{4}$, filtered, and concentrated. The residue $(480 \mathrm{mg})$ was purified by silica gel column chromatography (hexane/AcOEt) to give the ester $\mathbf{1 3}$ (423 mg, 98\%) as colorless needles; $\mathrm{mp} 154.5 \sim 155.0^{\circ} \mathrm{C}$ (hexane $/ \mathrm{CH}_{2} \mathrm{Cl}_{2}$ ); $[\alpha]_{\mathrm{D}}^{20}+59.0\left(c 1.0, \mathrm{CHCl}_{3}\right.$ ); IR (ATR) $\mathrm{cm}^{-1}$ : 3335, 2955, 2359, 1735, 1696, 1533, 1194, 1132, 1073, 1038; ${ }^{1} \mathrm{H}$ NMR $\left(300 \mathrm{MHz}, \mathrm{CDCl}_{3}\right) \delta 7.22 \sim 7.41$ $(10 \mathrm{H}, \mathrm{m}), 5.43 \sim 5.62(2 \mathrm{H}, \mathrm{m}), 5.11(2 \mathrm{H}, \mathrm{br} \mathrm{s}), 4.73 \sim 5.03$ $(3 \mathrm{H}, \mathrm{m}), 3.64(2 \mathrm{H}, \mathrm{s}), 2.42(1 \mathrm{H}, \mathrm{ddd}, J=12.6,10.2,2.4 \mathrm{~Hz})$, $1.50 \sim 1.68(1 \mathrm{H}, \mathrm{m}), 1.26(3 \mathrm{H}, \mathrm{brd}, J=6.6 \mathrm{~Hz}), 1.11(3 \mathrm{H}, \mathrm{d}$, $J=6.3 \mathrm{~Hz}), 0.96 \sim 1.22(4 \mathrm{H}, \mathrm{m}), 0.80(3 \mathrm{H}, \mathrm{t}, J=6.9 \mathrm{~Hz}) ;{ }^{13} \mathrm{C}$ NMR $\left(75 \mathrm{MHz}, \mathrm{CDCl}_{3}\right) \delta 172.6,170.1,170.0,155.4$, $135.9,133.1,129.1,128.6,128.5,128.2,128.0,127.4$, 75.9, 74.1, 70.9, 67.2, 54.7, 49.9, 41.4, 29.0, 27.8, 22.2, 17.5, 14.6, 13.7; MS (CI) $m / z 526\left([\mathrm{M}+\mathrm{H}]^{+}\right.$, base peak), 418, 381, 291, 236, 155, 91; HRMS (CI) $\mathrm{m} / z 526.2422$ $\left([\mathrm{M}+\mathrm{H}]^{+}\right)\left(526.2440\right.$ calcd for $\left.\mathrm{C}_{29} \mathrm{H}_{36} \mathrm{NO}_{8}\right)$.

\section{2-(Benzyloxy)-3-methanamidobenzoic Acid (14)}

2-Hydroxy-3-nitrobenzoic Acid to Benzyl 2-(Benzyloxy)3-nitrobenzoate

To a solution of 2-hydroxy-3-nitrobenzoic acid $(5.16 \mathrm{~g}$, $27.3 \mathrm{mmol})$ in THF $(43 \mathrm{ml})$ were added triphenylphosphine $(21.2 \mathrm{~g}, 81.9 \mathrm{mmol})$ and benzyl alcohol $(8.5 \mathrm{ml}, 81.9 \mathrm{mmol})$ at r.t., and then a solution of diethyl azodicarboxylate $(13 \mathrm{ml}, 81.9 \mathrm{mmol})$ in THF $(28 \mathrm{ml})$ was added dropwise to the mixture over 2 hours. The reaction mixture was quenched with the addition of saturated aqueous $\mathrm{NaHCO}_{3}$, and the resulting mixture was extracted with AcOEt. The organic extracts were dried over $\mathrm{Na}_{2} \mathrm{SO}_{4}$, filtered, and concentrated. The residue $(52 \mathrm{~g})$ was purified by silica gel column chromatography (hexane/EtOAc) to give the crude benzyl 2-(benzyloxy)-3-nitrobenzoate (10.3 g).

Benzyl 2-(Benzyloxy)-3-nitrobenzoate to Benzyl 3-Amino2-(benzyloxy)benzoate

A mixture of crude benzyl 2-(benzyloxy)-3-nitrobenzoate $(5 \mathrm{~g}, c a .13 .8 \mathrm{mmol})$ and iron powder $(4.6 \mathrm{~g}, 82.6 \mathrm{mmol})$ in $50 \% \mathrm{EtOH}$ aq. $(40 \mathrm{ml}, 1 / 1, \mathrm{v} / \mathrm{v})$ was heated at $100^{\circ} \mathrm{C}$, and then conc. $\mathrm{H}_{2} \mathrm{SO}_{4}$ in $50 \%$ EtOH aq. $(287 \mu \mathrm{l} / 1.38 \mathrm{ml})$ was added. After refluxing for 2 hours, the resulting mixture was treated with saturated aqueous $\mathrm{NaHCO}_{3}$ and then filtered through a pad of Celite. The filtrate was extracted with EtOAc, and the organic extracts were dried over $\mathrm{Na}_{2} \mathrm{SO}_{4}$, filtered, and concentrated to give the crude benzyl 3-amino-2-(benzyloxy)benzoate (4.38 g).

Benzyl 3-Amino-2-(benzyloxy)benzoate to 3-Amino-2(benzyloxy)benzoic Acid

To a solution of benzyl 3-amino-2-(benzyloxy)benzoate $(2.4 \mathrm{~g}, c a .7 .2 \mathrm{mmol})$ in $50 \% \mathrm{EtOH}$ aq. $(30 \mathrm{ml}, 1 / 1, \mathrm{v} / \mathrm{v})$ were added $6 \mathrm{M} \mathrm{NaOH}(4.8 \mathrm{ml}, 14.8 \mathrm{mmol})$ at $0^{\circ} \mathrm{C}$. After stirring at r.t. for 20 hours, the mixture was diluted with $\mathrm{H}_{2} \mathrm{O}$ and EtOAc. The organic layer was extracted with $0.5 \mathrm{M} \mathrm{NaOH}$ and the combined aqueous layers were washed with ether. After acidifing with $2 \mathrm{M} \mathrm{HCl}$, the acidic aqueous layer was extracted with AcOEt. The combined organic extracts were washed with a phosphate buffer $(\mathrm{pH}$ 6.8), dried over $\mathrm{Na}_{2} \mathrm{SO}_{4}$, filtered, and concentrated to give 3-amino-2-(benzyloxy)benzoic acid (1.74 g, 93\%, 3 steps from 2-hydroxy-3-nitrobenzoic acid).

\section{3-Amino-2-(benzyloxy)benzoic Acid to $\mathbf{1 4}$}

To a solution of formic acid $(235 \mu \mathrm{l}, 6.15 \mathrm{mmol})$ in $\mathrm{CH}_{2} \mathrm{Cl}_{2}$ $(5 \mathrm{ml})$ was added $N, N^{\prime}$-dicyclohexylcarbodiimide $(1.3 \mathrm{~g}$ $6.15 \mathrm{mmol}$ ) at $0^{\circ} \mathrm{C}$. After stirring at $0^{\circ} \mathrm{C}$ for 0.5 hours, a solution of 3-amino-2-(benzyloxy)benzoic acid $(1.07 \mathrm{~g}$ $4.1 \mathrm{mmol})$ in $\mathrm{CH}_{2} \mathrm{Cl}_{2}(20 \mathrm{ml})$ was added dropwise to the mixture over 20 minutes, and the mixture was stirred for 1 hour at r.t. The reaction mixture was filtered and the filtrate was concentrated. The residue was diluted with ether and extracted with aqueous $\mathrm{NaHCO}_{3}$. The combined aqueous layers were washed with ether and acidified with $6 \mathrm{M} \mathrm{HCl}$. The acidic aqueous layer was extracted with EtOAc, and the organic extracts were dried over $\mathrm{Na}_{2} \mathrm{SO}_{4}$, filtered, and concentrated to give formylamide $\mathbf{1 4}(1.04 \mathrm{~g}, 93 \%)$ as a brown powder (rotamer mixture); mp $170.0 \sim 171.5^{\circ} \mathrm{C}$; IR $(\mathrm{KBr}) \mathrm{cm}^{-1}$ : 3346, 2769, 1700, 1645; ${ }^{1} \mathrm{H}$ NMR $(200 \mathrm{MHz}$, DMSO $\left.d_{6}\right) \delta: 13.0(1 \mathrm{H}, \mathrm{br} \mathrm{s}), 9.71,9.71$ (total integr. $1 \mathrm{H}, \mathrm{s}$, br d, $J=11.8 \mathrm{~Hz}$ ), 8.51, 8.35, 8.32, $7.61 \sim 7.12$ (total integr. $9 \mathrm{H}$, br d, $J=10.6 \mathrm{~Hz}, \mathrm{~d}, J=1.0 \mathrm{~Hz}, \mathrm{dd}, J=8.0,1.6 \mathrm{~Hz}, \mathrm{~m}$ ), 4.96, 4.93 (total integr. 2H, s, s); MS (EI) $m / z 227\left(\mathrm{M}^{+}\right.$), 181, 135, 106, 91 (base peak); HMRS (EI) $m / z 271.0837$ (271.0844 calcd for $\mathrm{C}_{15} \mathrm{H}_{13} \mathrm{NO}_{4}$ ).

\section{$(2 R, 3 S, 6 S, 7 R, 8 R)-3-[2-(B e n z y l o x y)-3-$ methanamidophenylamido]-8-butyl-2,6-dimethyl-4,9- dioxo-1,5-dioxonan-7-yl 2-Phenylethanoate (15)}

A mixture of 13 ( $424 \mathrm{mg}, 0.81 \mathrm{mmol}$ ) and $\mathrm{Pd} / \mathrm{C}$ (catalytic amount) in THF $(2.5 \mathrm{ml})$ was stirred under $\mathrm{H}_{2}$ atmosphere for 2 hours in the dark. The mixture was filtered through a pad of Celite, and the filtrate was concentrated. To a solution of the residual oil in DMF $(2.5 \mathrm{ml})$ were added 3formamidesalicylic acid derivative $\mathbf{1 4}(395 \mathrm{mg}, 1.5 \mathrm{mmol})$, WSC (239 mg, $1.3 \mathrm{mmol})$, HOBt (223 mg, $1.5 \mathrm{mmol})$, and $\operatorname{NMM}(625 \mu \mathrm{l}, 5.7 \mathrm{mmol})$ successively at r.t. After stirring 
for 24 hours, the reaction mixture was quenched with the addition of saturated aqueous $\mathrm{NaHCO}_{3}$, and the resulting mixture was extracted with EtOAc. The organic extracts were dried over $\mathrm{MgSO}_{4}$, filtered, and concentrated. The residue $(502 \mathrm{mg})$ was purified by silica gel column chromatography (hexane/EtOAc) to give the amide $\mathbf{1 5}$ (391 mg, 77\%, 2 steps) as a colorless solid (rotamer mixture); $\mathrm{mp} 137.5 \sim 139.5^{\circ} \mathrm{C} ;[\alpha]_{\mathrm{D}}^{24}+34.2\left(c 1.0, \mathrm{CHCl}_{3}\right)$; IR (ATR) $\mathrm{cm}^{-1}: 3302,2955,1739,1686,1645,1518,1189$, 1129,$1024 ;{ }^{1} \mathrm{H}$ NMR $\left(300 \mathrm{MHz}, \mathrm{CDCl}_{3}\right) \delta 8.54$ and 8.42 (total integr. $1 \mathrm{H}$, br d, $J=11.1 \mathrm{~Hz}$ and dd, $J=8.1,1.5 \mathrm{~Hz}$ ), $8.02 \sim 8.20$ and $7.20 \sim 7.60$ (total integr. $14 \mathrm{H}, \mathrm{m}$ and $\mathrm{m}$ ), 7.81 and 7.72 (total integr. $1 \mathrm{H}, \mathrm{brd}, J=6.3 \mathrm{~Hz}$ and dd, $J=8.1,1.8 \mathrm{~Hz}$ ), 5.66 and 5.64 (total integr. $1 \mathrm{H}$, quintet, $J=7.2 \mathrm{~Hz}$ and quintet, $J=7.2 \mathrm{~Hz}$ ), 5.31 and 5.28 (total integr. $1 \mathrm{H}, \mathrm{t}, J=7.8 \mathrm{~Hz}$ and $\mathrm{t}, J=9.6 \mathrm{~Hz}), 5.15(1 \mathrm{H}, \mathrm{d}$, $J=11.4 \mathrm{~Hz}), \quad 5.03(1 \mathrm{H}, \quad \mathrm{t}, \quad J=9.9 \mathrm{~Hz}), 4.85(1 \mathrm{H}, \quad \mathrm{d}$, $J=11.4 \mathrm{~Hz}), 4.80 \sim 4.96(2 \mathrm{H}, \mathrm{m}), 3.66(2 \mathrm{H}, \mathrm{s}), 2.45(1 \mathrm{H}, \mathrm{t}$, $J=9.9 \mathrm{~Hz}), 1.50 \sim 1.66(1 \mathrm{H}, \mathrm{m}), 1.25(3 \mathrm{H}, \mathrm{d}, J=6.6 \mathrm{~Hz})$, $1.16(3 \mathrm{H}, \mathrm{d}, J=6.0 \mathrm{~Hz}), 0.98 \sim 1.30(5 \mathrm{H}, \mathrm{m}), 0.81(3 \mathrm{H}, \mathrm{t}$, $J=6.6 \mathrm{~Hz}) ;{ }^{13} \mathrm{C}$ NMR $\left(75 \mathrm{MHz}, \mathrm{CDCl}_{3}\right) \delta 172.7,172.3$, $170.2,170.0,164.7,164.3,161.1,158.6,146.6,146.0$, $135.1,133.1,134.4,133.1,131.3,129.3,129.1,129.0$, $128.8,128.7,127.4,126.3,126.1,125.6,125.4,124.8$, 121.7, 78.6, 78.2, 76.0, 71.0, 70.9, 54.0, 50.0, 41.5, 29.1, 27.9, 22.2, 17.6, 15.0, 13.7; MS (CI) $m / z 645\left([\mathrm{M}+\mathrm{H}]^{+}\right)$, 555, 418, 355, 337, 291 (base peak), 228, 195, 155; HRMS (CI) $m / z \quad 645.2792\left([\mathrm{M}+\mathrm{H}]^{+}\right) \quad(645.2811$ calcd for $\mathrm{C}_{36} \mathrm{H}_{41} \mathrm{~N}_{2} \mathrm{O}_{9}$ ).

\section{Antimycin $\mathbf{A}_{9}$}

A mixture of 15 (358 $\mathrm{mg}, 0.56 \mathrm{mmol})$ and $\mathrm{Pd} / \mathrm{C}$ (catalytic amount) in EtOAc $(3 \mathrm{ml})$ was stirred under $\mathrm{H}_{2}$ atmosphere for 2 hours. The mixture was filtered through a pad of Celite, and the filtrate was concentrated. The residue $(295 \mathrm{mg}$ ) was purified by silica gel column chromatography (hexane/EtOAc) to give antimycin $\mathrm{A}_{9}(284 \mathrm{mg}, 89 \%)$ as a pale yellow solid (rotamer mixture); $\mathrm{mp} 151.0 \sim 152.0^{\circ} \mathrm{C}$; $[\alpha]_{\mathrm{D}}^{22}+82.1(c 0.17, \mathrm{MeOH})$; IR (ATR) $\mathrm{cm}^{-1}: 3372,2957$, $2872,2360,2341,1745,1530,1364,1182 ;{ }^{1} \mathrm{H}$ NMR $\left(600 \mathrm{MHz}, \mathrm{CDCl}_{3}\right) \delta 12.61$ and 12.47 (total integr. $1 \mathrm{H}, \mathrm{s}$ and s), 8.09 and 8.50 (total integr. $1 \mathrm{H}, \mathrm{d}, J=11.0 \mathrm{~Hz}$ and br s), $8.55(1 \mathrm{H}, \mathrm{d}, J=10.2 \mathrm{~Hz}), 8.11$ and 7.83 (total integr. $1 \mathrm{H}$, brs and brd, $J=10.2 \mathrm{~Hz}), 7.32 \sim 7.38(2 \mathrm{H}, \mathrm{m})$, $7.26 \sim 7.32(3 \mathrm{H}, \mathrm{m}), 7.24(1 \mathrm{H}, \mathrm{dd}, J=8.0,0.8 \mathrm{~Hz}), 7.10$ and 6.91 (total integr. $1 \mathrm{H}, \mathrm{d}, J=7.7 \mathrm{~Hz}$ and d, $J=7.7 \mathrm{~Hz}$ ), 6.90 $(1 \mathrm{H}, \mathrm{t}, J=8.2 \mathrm{~Hz}), 5.75$ and 5.71 (total integr. $1 \mathrm{H}, \mathrm{q}$, $J=6.9 \mathrm{~Hz}$ and q, $J=7.1 \mathrm{~Hz}$ ), 5.32 and 5.27 (total integr. $1 \mathrm{H}$, t, $J=7.7 \mathrm{~Hz}$ and t, $J=7.4 \mathrm{~Hz}$ ), 5.09 and 5.05 (total integr. $1 \mathrm{H}, \mathrm{t}, J=10.2 \mathrm{~Hz}$ and t, $J=10.2 \mathrm{~Hz}), 4.93(1 \mathrm{H}, \mathrm{dq}, J=6.6$, $6.3 \mathrm{~Hz}), 3.67$ (2H, s), 2.49 (1H, ddd, $J=13.2,10.2,2.7 \mathrm{~Hz}$ ),
$1.54 \sim 1.62(1 \mathrm{H}, \mathrm{m}), 1.30(3 \mathrm{H}, \mathrm{d}, J=6.6 \mathrm{~Hz}), 1.15(3 \mathrm{H}, \mathrm{d}$, $J=6.3 \mathrm{~Hz}), 1.10 \sim 1.24(4 \mathrm{H}, \mathrm{m}), 1.00 \sim 1.08(1 \mathrm{H}, \mathrm{m}), 0.81$ $(3 \mathrm{H}, \mathrm{t}, J=7.1 \mathrm{~Hz}) ;{ }^{13} \mathrm{C}$ NMR $\left(150 \mathrm{MHz}, \mathrm{CDCl}_{3}\right) \delta 172.7$, $170.2,170.0,169.3,159.2,150.7,133.0,129.2,128.8$, $127.5,127.4,124.7,120.0,118.9,112.5,75.8,74.7,70.8$, 53.5, 50.1, 41.5, 29.1, 27.9, 22.3, 17.6, 14.9, 13.7; MS (FAB) $m / z 577\left([\mathrm{M}+\mathrm{Na}]^{+}\right), 555\left([\mathrm{M}+\mathrm{H}]^{+}\right), 413,391,265$, 154, 55 (base peak), 41; HRMS (FAB) $\mathrm{m} / z 555.2314$ $\left([\mathrm{M}+\mathrm{H}]^{+}\right)\left(555.2342\right.$ calcd for $\left.\mathrm{C}_{29} \mathrm{H}_{35} \mathrm{~N}_{2} \mathrm{O}_{9}\right)$.

Acknowledgment We thank Professor Satoshi Ōmura and Kazuro Shiomi (Institute for Life Sciences, Kitasato University and the Kitasato Institute, Japan) for providing the ${ }^{1} \mathrm{H}$ and ${ }^{13} \mathrm{C}$ spectra of authentic (+)-antimycin $\mathrm{A}_{9}$. This work was supported partially by a Grant-in-Aid for Scientific Research (C) from MEXT (the Ministry of Education, Culture, Sports, Science and Technology of Japan). We are also thankful to MEXT.HAITEKU, 2003 2007.

\section{References}

1. a) Bycroft DW. Dictionary of antibiotics and related substances. pp. 128, Chapman and Hall, London (1988) b) Shiomi K, Hatae K, Hatano H, Matsumoto A, Takahashi Y, Jiang CL, Tomoda H, Kobayashi S, Tanaka H, Ōmura S. A new antibiotic, Antimycin $\mathrm{A}_{9}$, produced by Streptomyces sp. K01-0031. J Antibiot 58: 74-78 (2005)

c) Hosotani N, Kumagai K, Nakagawa H, Shimatani T, Saji I. Antimycins $\mathrm{A}_{10} \sim \mathrm{A}_{16}$, seven new antimycin antibiotics produced by Streptomyces spp. SPA-10191 and SPA-8893. J Antibiot 58: 460-467 (2005)

d) Chen G, Lin B, Lin Y, Xie F, Lu W, Fong WF. A new fungicide produced by a Streptomyces sp. GAAS7310. J Antibiot 58: 519-522 (2005) and references cited therein.

2. a) Abidi SL. High-performance liquid chromatographic separation of subcomponents of antimycin A. J Chromatogr 447: 65-79 (1988)

b) Abidi SL. Reversed-phase thin-layer chromatography of homologues of antimycin A and related derivatives. J Chromatogr 464: 453-458 (1989)

3. a) Kinoshita M, Wada M, Aburaki S, Umezawa S. Total synthesis of Antimycin A . J Antibiot 24: 724-726 (1971)

b) Kondo $H$, Oritani $T$, Kiyota $H$. Synthesis and antimicrobial activity of unnatural (-)-antimycin $\mathrm{A}_{3}$ and its analog. Heterocyclic Commun 6: 211-214 (2000)

c) Tsunoda T, Nishii T, Yoshizuka M, Yamasaki C, Suzuki T, Itô $\mathrm{S}$. A total synthesis of (-)-antimycin $\mathrm{A}_{3 \mathrm{~b}}$. Tetrahedron Lett 41: 7667-7671 (2000)

d) Nishii T, Suzuki S, Yoshida K, Arakaki K, Tsunoda T. Total synthesis of the $(+)$-antimycin $\mathrm{A}_{3}$ family: structure elucidation of $(+)$-antimycin $\mathrm{A}_{3 \mathrm{a}}$. Tetrahedron Lett 44: 7829-7832 (2003)

e) Wu Y, Yang YQ. An expeditious enantioselective 
synthesis of antimycin $\mathrm{A}_{3 \mathrm{~b}}$. J Org Chem 71: 4296-4301 (2006)

4. When the aldol reaction of $\mathbf{2}$ and $\mathbf{3}$ was carried out under the original conditions, none of the desired compound was obtained.

5. The stereochemistry of the minor adduct was presumed to be $2 S, 3 S$.

6. Oppolzer W, Blagg J, Rodriguez I, Walther E. Bornane sultam-directed asymmetric synthesis of crystalline, enantiomerically pure syn aldols. J Am Chem Soc 112: 2767-2772 (1990)

7. Oppolzer W, Lienard P. Non-destructive cleavage of $\mathrm{N}$ - acylsultams under neutral conditions: Preparation of enantiomerically, pure Fmoc-protected a-amino acids. Helv Chim Acta 75: 2572-2582 (1992)

8. Wasserman HH, Gambale RJ. Synthesis of (+)-antimycin $\mathrm{A}_{3}$. Use of the oxazole ring in protecting and activating functions. J Am Chem Soc 107: 1423-1424 (1985)

9. Inanaga J, Hirata K, Saeki H, Katsuki T, Yamaguchi M. A rapid esterification by means of mixed anhydride and its application to large-ring lactonization. Bull Chem Soc Jpn 52: 1989-1993 (1979)

10. This is the first example of lactonization of pyridinethiol ester using (CuOTf $)_{2} \cdot \mathrm{PhH}$. 